\title{
Optic axis-driven new horizons for hyperbolic metamaterials
}

\author{
Allan D. Boardman ${ }^{1, *}$, Peter Egan ${ }^{1}$, and Martin $\mathrm{McCall}^{2}$ \\ 1 Joule Physics Laboratory, Materials \& Physics Research Centre, University of Salford, Greater Manchester M5 4WT, UK \\ 2 Blackett Laboratory, Imperial College, Prince Consort Road, London SW7 2AZ, UK
}

Received 1 September 2015 / Accepted 28 October 2015

\begin{abstract}
The broad assertion here is that the current hyperbolic metamaterial world is only partially served by
investigations that incorporate only some limited version of anisotropy. Even modest deviations of the optic axis from
the main propagation axis lead to new phase shifts, which not only compete with those created by absorption but end
up dominating them. Some progress has been attempted in the literature by introducing the terms "asymmetric hyper-
bolic media", but it appears that this kind of asymmetry only involves an optic axis at an angle to the interface of a
uniaxial crystal. From a device point of view, many new prospects should appear and the outcomes of the investiga-
tions presented here yield a new general theory. It is emphasised that the orientation of the optic axis is a significant
determinant in the resulting optical properties. Whereas for conventional anisotropic waveguides homogeneous prop-
agating waves occur over a limited range of angular dispositions of the optic axis it is shown that for a hyperbolic
guide a critical angular setting exists, above which the guided waves are always homogeneous. This has significant
implications for metawaveguide designs. The resulting structures are more tolerant to optic axis misalignment.
\end{abstract}
Key words: Metamaterial, Hyperbolic, Uniaxial, Optic axis.

\section{Introduction}

The interest in calcite (Iceland spar) dates back to 1669 and the work of Bartholin [1]. This means that the theory of propagation of electromagnetic waves in such uniaxial anisotropic crystals has naturally been of interest for some considerable time. Indeed, the early investigations are extremely useful in forming a background and vital points of contact for the creation of new horizons in the global surge of interest in what has become known as hyperbolic [2] non-magnetic metamaterial. The current hyperbolic metamaterial world is only partially served by investigations that incorporate some limited version of anisotropy. In order to develop new pathways, it should first of all be noted that readily available uniaxial non-metamaterial crystals, like calcite, have a direction around which rotational symmetry is apparent. This direction is called the optic axis and, given a rectangular coordinate system, it is usually set to lie along the $z$-axis, which in the model presented here is going to be the direction of wave propagation. Adopting this step leads to the permittivity tensor [3] emerging in diagonal form, possessing equal $x$ and $y$ components that are unequal to the component associated with the $z$-direction. Hyperbolic metamaterials have the same $x$ and $y$ tensor properties but possess a negative $z$-component. A completely general

*e-mail: a.d.boardman@salford.ac.uk approach to the constructive manipulation of the optic axis position in uniaxial crystals is required if a proper description of any wave propagation within them is to be achieved. In this context, as will be emphasized here, there is a need to recognise clearly the dramatic role of anisotropy in uniaxial metamaterial investigations, as was pointed out some years ago [4].

In order to set up a full investigation of how the orientation of the optic axis affects wave propagation in hyperbolic metamaterials, it is efficient to focus upon two broad areas. Area one includes the interrogation of a slab of metamaterial, involving the transmission of light through an interface, or a number of interfaces. Area two invokes the paramount role of the optic axis when guided waves are being generated, and it is this case that will be studied here, where it will be shown that for a hyperbolic film, in contrast to the usual non-metamaterial anisotropic guides, substantial changes in the character of guided waves occur, presenting the opportunity for novel device design.

In general, there are off-diagonal permittivity tensor elements to be considered when controlling the optic axis direction. These elements can often be brought in unintentionally at experimental level when trying to compare measurements with simulations. This is emphasised in the work by Starodubtsev [4], in which he writes 


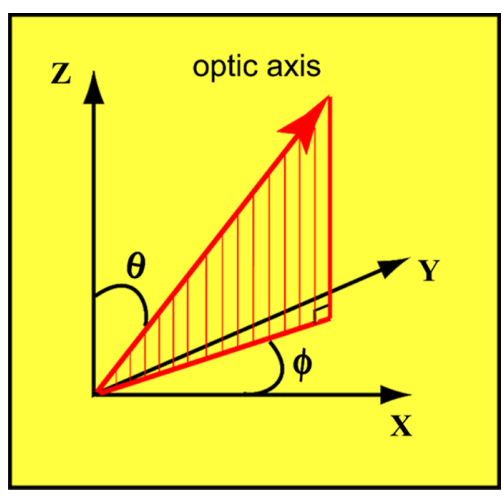

Figure 1. For a rectangular coordinate system $(x, y, z)$, the general position of the optic axis in a uniaxial crystal can be set at an angle $\theta$ to the $z$-axis. This axis then has a projection on the $(x, y)$ plane, which is at angle $\phi$ to the $x$-axis.

"even small optical anisotropy is a much greater enemy of the perfect lens than absorption".

Thus, even modest deviations of the optic axis from the main $z$-axis leads to new phase shifts, which not only compete with those created by absorption but may end up dominating them. Some progress has been attempted by introducing the term "asymmetric hyperbolic media" [5, 6], but it appears that this kind of asymmetry only involves an optic axis at a certain angle to the interface of a uniaxial crystal. Here, a full investigation is made of the effect that the optic axis direction has on the existence of pure hybrid homogeneous waves in a wave guide consisting of a hyperbolic film, an asymmetric substrate with all positive permittivity tensor elements, and an air cover.

\section{The optic axis}

The historic, classic work of Knoesen, Gaylord and Moharam [7] on non-metamaterial anisotropic uniaxial waveguides introduces the geometrical concept of an optic axis in the context of a slab waveguide. It is an attractive anisotropic design, which is why it is adopted here in order to illustrate the new metamaterial concepts to be introduced in this investigation. It does not limit the generality of the overall conclusions and, for the moment, all three components of the wave number, related to right angle Cartesian coordinates will be considered.

The classic diagonal form of the permittivity tensor for a uniaxial, non-magnetic crystal when the optic axis lies along the $z$-axis is given by

$$
\bar{\varepsilon}=\left(\begin{array}{ccc}
\varepsilon_{1} & 0 & 0 \\
0 & \varepsilon_{2} & 0 \\
0 & 0 & \varepsilon_{3}
\end{array}\right) .
$$

Referring to Figure 1, the general direction of the optic axis is described through the angles $\theta$ and $\phi$, where $\theta$ is the angle that the optic axis makes with the $z$-axis, and $\phi$ is the angle between the projection of the optic axis onto the $x y$-plane measured from the $x$-axis (Figure 1).
In order to take into account a variation in the orientation of the optic axis, the transformation matrix

$$
\mathbf{T}=\left(\begin{array}{ccc}
\sin \phi & \cos \theta \cos \phi & \sin \theta \cos \phi \\
-\cos \phi & \sin \phi \cos \theta & \sin \theta \sin \phi \\
0 & -\sin \theta & \cos \theta
\end{array}\right)
$$

can be used to find the elements of the general permittivity tensor,

$$
\varepsilon=\left(\begin{array}{ccc}
\varepsilon_{x x} & \varepsilon_{x y} & \varepsilon_{x z} \\
\varepsilon_{y x} & \varepsilon_{y y} & \varepsilon_{y z} \\
\varepsilon_{z x} & \varepsilon_{z y} & \varepsilon_{z z}
\end{array}\right)
$$

in the usual way, using $\boldsymbol{\varepsilon}=\mathbf{T} \bar{\varepsilon} \mathbf{T}^{-1}$. In the lossless cases being investigated, the tensor is Hermitian so that $\varepsilon_{i j}=\varepsilon_{j i}^{*}$. Thus, for real elements, the matrix is symmetric, $\varepsilon_{i j}=\varepsilon_{j i}$ and the elements are given by [8]

$$
\begin{gathered}
\varepsilon_{x x}=\varepsilon_{1} \sin ^{2} \phi+\left(\varepsilon_{1} \cos ^{2} \theta+\varepsilon_{3} \sin ^{2} \theta\right) \cos ^{2} \phi, \\
\varepsilon_{x y}=\varepsilon_{y x}=\left(\varepsilon_{3}-\varepsilon_{1}\right) \sin \phi \cos \phi \sin ^{2} \theta, \\
\varepsilon_{x z}=\varepsilon_{z x}=\left(\varepsilon_{3}-\varepsilon_{1}\right) \cos \phi \cos \theta \sin \theta, \\
\varepsilon_{y y}=\varepsilon_{1} \cos ^{2} \phi+\left(\varepsilon_{1} \cos ^{2} \theta+\varepsilon_{3} \sin ^{2} \theta\right) \sin ^{2} \phi, \\
\varepsilon_{y z}=\left(\varepsilon_{3}-\varepsilon_{1}\right) \sin \phi \cos \theta \sin \theta, \\
\varepsilon_{z z}=\varepsilon_{1} \sin ^{2} \theta+\varepsilon_{3} \cos ^{2} \theta,
\end{gathered}
$$

In special cases, for example when $\phi=0^{\circ}$ and the optic axis lies in $x z$-plane [9], or when $\theta=90^{\circ}$ and the optic axis lies in the $x y$-plane [10], the relevant permittivity tensor can be found using the appropriate values of $\theta$ and $\phi$ in equation (3).

For wave propagation, a useful characteristic is the dimensionless wave number with components $\kappa_{i}=\frac{c k_{i}}{\omega}$, where $i=x, y$ or $z, c$ is the velocity of light in free-space and $\omega$ is the angular frequency. For the case when all the diagonal tensor elements are positive, two surfaces in $\left(\kappa_{x}, \kappa_{y}, \kappa_{z}\right)$ space will appear for a given frequency. One is that of an ellipsoid and the other is that of a sphere. The former is classically associated with extraordinary modes where, in general, the power flow and phase velocity direction are not parallel. The sphere is associated with ordinary modes where the power flow and the phase velocity directions are the same. For a metamaterial described by a diagonal dielectric tensor with two equal positive elements, and one negative element, the ordinary wave sphere still emerges but the ellipsoid opens up into a hyperboloid. Figure 2 demonstrates this for the case when the optic axis lies along the $z$-axis [11].

Following the magnificent lead, set many years ago by the rigorous and pioneering general work of Knoesen et al. [7], and some pioneering work on beam propagation by Fleck and Feit [12], the discussion presented below will focus upon a lossfree, planar, linear uniaxial waveguide (Figure 3), where the subscripts $s, f$ and $c$ are used to represent the substrate, film and cover respectively.

In this investigation the planar guide has a hyperbolic metamaterial central film with $\varepsilon_{3 f}<0$, an anisotropic substrate with $\varepsilon_{3 s}>0$, and an isotropic cover, assumed for the moment 


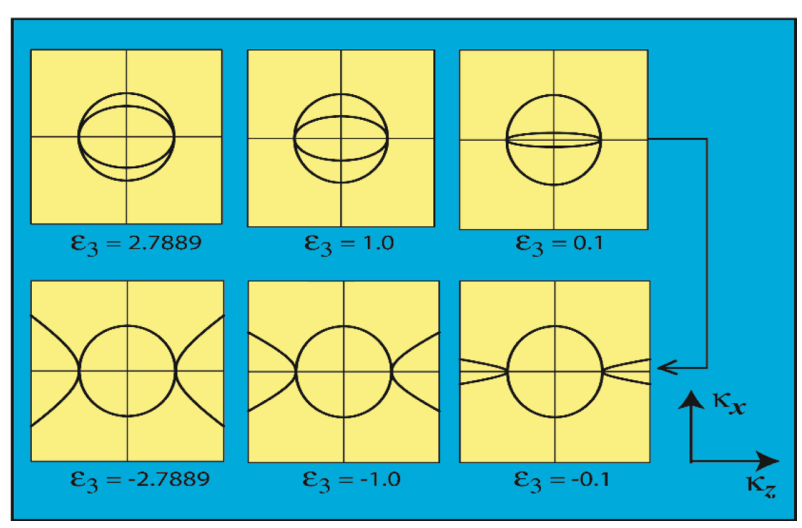

Figure 2. Cross-sections through the $\left(\kappa_{x}, \kappa_{z}\right)$-plane in wavenumber space when $\kappa_{y}=0$ and the optic axis lies along the $\kappa_{z}$-axis. The figure shows the transition from anisotropic (ellipse) to hyperbolic as $\varepsilon_{3}$ changes in incremental steps from a positive to a negative value. The element $\varepsilon_{1}=\varepsilon_{2}=4.0$.

to be air. The adoption of losses can be introduced at a later stage of any device design but it is not a paramount issue here since hyperbolic metamaterials, unlike double-negative metamaterials, are not resonant. The model here has an optic axis that is permitted to have any orientation, but is the same throughout for the film, the substrate and the cover [7]. Uncoupling of the TE and TM modes occurs when the angle $\phi=0^{\circ}$. Otherwise, the waves are hybrid and will be highlighted in terms of pure guided homogeneous modes, pure guided inhomogeneous modes, or leaky guided modes [7]. A clarification of the homogeneous modes is that these waves have constant planar phase fronts, and are totally guided with constant amplitudes. Inhomogeneous waves, on the other hand, are characterised by planar phase fronts with non-constant amplitudes. Leaky modes are not totally guided by a slab waveguide. The main thrust in this paper is in the effect that the orientation of the optic axis has on the existence of pure hybrid homogeneous modes. The term "anisotropic" will be used when all the elements of the permittivity tensor are positive, and, specifically, when $\varepsilon_{1}=\varepsilon_{2}>0 ; \varepsilon_{3}>0 \neq \varepsilon_{1}$. The expression "hyperbolic" will be used to mean anisotropic, with $\varepsilon_{1}=\varepsilon_{2}>0$ and $\varepsilon_{3}<0$.

In the absence of attenuation, the values of $\kappa_{z}$ (the component of the wavenumber in the propagating direction) are real, and, in the case of an anisotropic film, are restricted by the permittivity of the isotropic cover, and the permittivity tensors of the anisotropic substrate and the hyperbolic film. In the anisotropic film case, the maximum value that $\kappa_{z}$ is can take is $\sqrt{ } \boldsymbol{\varepsilon}_{\mathbf{1}}$. However, under certain orientations of the optic axis, to be determined later, this upper boundary no longer applies when the film is hyperbolic.

If the optic axis lies in the $x z$-plane, then the range of values of $\theta$ over which the important homogeneous waves propagate, is restricted to a specific range for both anisotropic and hyperbolic guides. For an anisotropic film, if the optic axis is also rotated so that angle $\phi$ has a positive value, then the range of $\theta$ over which homogeneous waves propagate decreases. In contrast, when using a hyperbolic metamaterial-based guide, increasing $\phi$ from $0^{\circ}$ results in an increase in the range of $\theta$

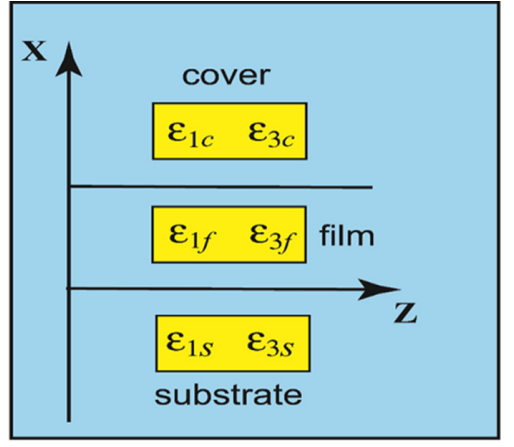

Figure 3. Planar, step-index, waveguide with guided waves propagating along $z$, for which the central film $(f)$ is a hyperbolic metamaterial. The cover $(c)$ and substrate $(s)$ can be purely isotropic, or anisotropic, with positive tensor elements.

over which pure hybrid homogeneous modes propagate until, at a certain critical value of $\phi$, these modes will propagate for all angles of $\theta$ between $0^{\circ}$ and $90^{\circ}$. At angles of $\phi$ greater than this critical angle, modes are possible with $\kappa_{z}^{2}>\varepsilon_{1 f}$ but these modes are hybrid inhomogeneous waves. This will be discussed in more detail later.

\section{Wave propagation and design windows}

First of all, consider a bulk non-magnetic uniaxial crystal, with a permittivity tensor $\varepsilon_{i j}$. This material will support plane waves of the form $\mathbf{A} e^{\mathrm{i}(\mathbf{k \bullet r}-\omega t)}$, where the complex wave vector amplitude is $\mathbf{A}$, and wave vector and position vector are, respectively, $\mathbf{k}=\left(k_{x}, k_{y}, k_{z}\right)$ and $\mathbf{r}=(x, y, z)$. Maxwell's equations then yield the following equation for the electric field components $\left(E_{x}, E_{y}, E_{z}\right)$.

$$
\left(\begin{array}{ccc}
k^{2}-k_{x}^{2}-\frac{\omega^{2}}{c^{2}} \varepsilon_{x x} & -k_{x} k_{y}-\frac{\omega^{2}}{c^{2}} \varepsilon_{x y} & -k_{x} k_{z}-\frac{\omega^{2}}{c^{2}} \varepsilon_{x z} \\
-k_{x} k_{y}-\frac{\omega^{2}}{c^{2}} \varepsilon_{y x} & k^{2}-k_{y}^{2}-\frac{\omega^{2}}{c^{2}} \varepsilon_{y y} & -k_{y} k_{z}-\frac{\omega^{2}}{c^{2}} \varepsilon_{y z} \\
-k_{x} k_{z}-\frac{\omega^{2}}{c^{2}} \varepsilon_{z x} & -k_{y} k_{z}-\frac{\omega^{2}}{c^{2}} \varepsilon_{y z} & k^{2}-k_{z}^{2}-\frac{\omega^{2}}{c^{2}} \varepsilon_{z z}
\end{array}\right)\left(\begin{array}{c}
E_{x} \\
E_{y} \\
E_{z}
\end{array}\right)=0
$$

or, in terms of the dimensionless wave vector $\kappa_{i}$ defined above

$$
\left(\begin{array}{ccc}
\kappa_{y}^{2}+\kappa_{z}^{2}-\varepsilon_{x x} & -\kappa_{x} \kappa_{y}-\varepsilon_{x y} & -\kappa_{x} \kappa_{z}-\varepsilon_{x z} \\
-\kappa_{x} \kappa_{y}-\varepsilon_{x y} & \kappa_{x}^{2}+\kappa_{z}^{2}-\varepsilon_{y y} & -\kappa_{y} \kappa_{z}-\varepsilon_{y z} \\
-\kappa_{x} \kappa_{z}-\varepsilon_{x z} & -\kappa_{y} \kappa_{z}-\varepsilon_{y z} & \kappa_{x}^{2}+\kappa_{y}^{2}-\varepsilon_{z z}
\end{array}\right)\left(\begin{array}{c}
E_{x} \\
E_{y} \\
E_{z}
\end{array}\right)=0
$$

In the planar waveguide (Figure 2), as is usual, it is assumed that there is no variation of the fields in the $y$-direction so that setting $\kappa_{y}=0$ in equation (5) yields the biquadratic

$$
\begin{gathered}
\kappa_{x}^{4} \varepsilon_{x x}+2 \kappa_{x}^{3} \kappa_{z} \varepsilon_{x z}+\kappa_{x}^{2}\left[\kappa_{z}^{2}\left(\varepsilon_{x x}+\varepsilon_{z z}\right)+\varepsilon_{x y}^{2}+\varepsilon_{x z}^{2}-\varepsilon_{x x} \varepsilon_{z z}-\varepsilon_{x x} \varepsilon_{y y}\right] \\
+2 \kappa_{x} \kappa_{z}\left[\kappa_{z}^{2} \varepsilon_{x z}+\varepsilon_{x y} \varepsilon_{y z}-\varepsilon_{x z} \varepsilon_{y y}\right]+\kappa_{z}^{4} \varepsilon_{z z}+\kappa_{z}^{2}\left(\varepsilon_{y z}^{2}+\varepsilon_{x z}^{2}-\varepsilon_{y y} \varepsilon_{z z}-\varepsilon_{x x} \varepsilon_{z z}\right) \\
+\varepsilon_{x x} \varepsilon_{y y} \varepsilon_{z z}+2 \varepsilon_{x y} \varepsilon_{y z} \varepsilon_{x z}-\varepsilon_{x x} \varepsilon_{y z}^{2}-\varepsilon_{y y} \varepsilon_{x z}^{2}-\varepsilon_{x y}^{2} \varepsilon_{z z}=0
\end{gathered}
$$



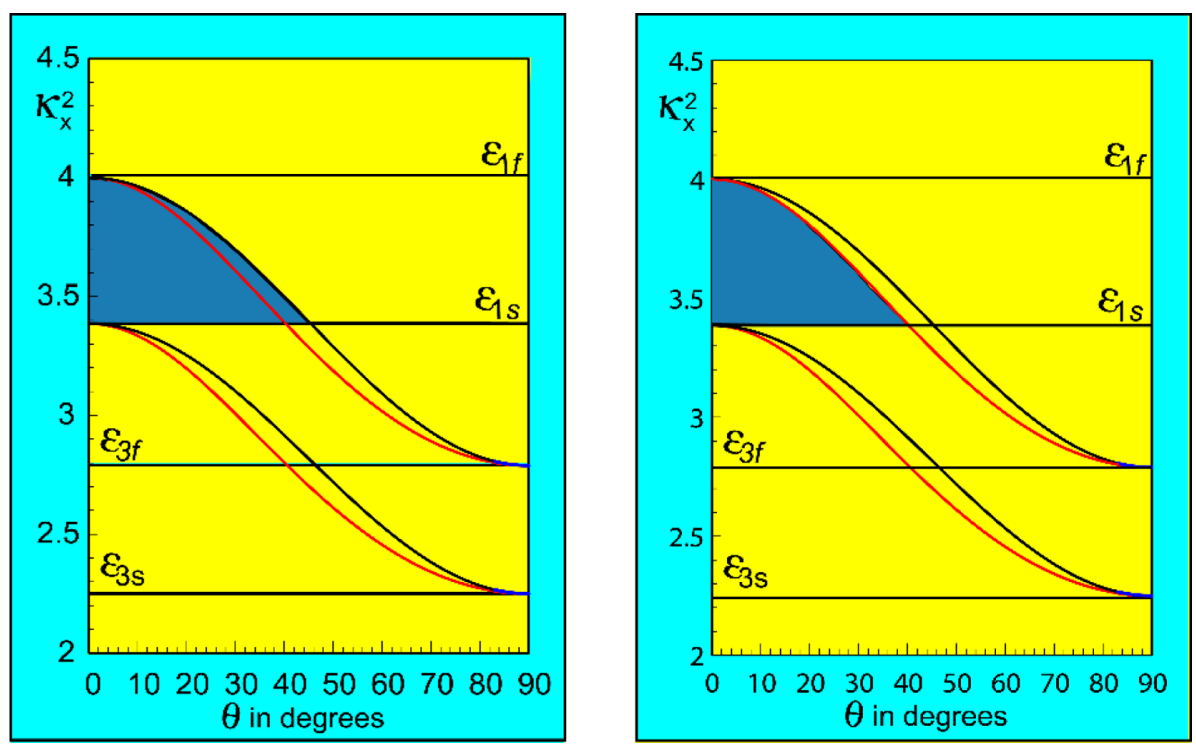

Figure 4. The change in the regions defining pure hybrid homogeneous waves for the anisotropic guide with all-positive diagonal tensor elements as the angles of orientation are changed. Values used: $\varepsilon_{1 s}=3.3856, \varepsilon_{3 s}=2.25, \varepsilon_{1 f}=4$ and $\varepsilon_{3 f}=2.7889$. The blue areas show how changing the value of $\phi$ from $\phi=0^{\circ}$ (the blue curves) to $\phi=90^{\circ}$ (the red curves) has little effect on the range of $\theta$ over which pure hybrid homogeneous modes are possible.

which, with judicious use of equations (3), reduces to

$$
\begin{aligned}
& \varepsilon_{x x} \kappa_{x}^{4}+2 \varepsilon_{x z} \kappa_{z} \kappa_{x}^{3}+\left[\kappa_{z}^{2}\left(\varepsilon_{x x}+\varepsilon_{z z}\right)-\varepsilon_{1}\left(\varepsilon_{x x}+\varepsilon_{3}\right)\right] \kappa_{x}^{2} \\
& +2 \varepsilon_{x z} \kappa_{z} \kappa_{x}\left(\kappa_{z}^{2}-\varepsilon_{1}\right)+\kappa_{z}^{4} \varepsilon_{z z}-\kappa_{z}^{2} \varepsilon_{1}\left(\varepsilon_{z z}+\varepsilon_{3}\right)+\varepsilon_{1}^{2} \varepsilon_{3}
\end{aligned}
$$

which factorises to give

$$
\kappa_{z}^{2}+\kappa_{x}^{2}-\varepsilon_{1}=0 ; \quad \varepsilon_{x x} \kappa_{x}^{2}+2 \kappa_{z} \kappa_{x} \varepsilon_{x z}+\kappa_{z}^{2} \varepsilon_{z z}-\varepsilon_{1} \varepsilon_{3}=0
$$

These equations show how the elliptic case, with only positive diagonal tensor elements, turns into the hyperbolic case as $\varepsilon_{3}$ becomes negative (see for example Figure 2). At this stage, it is convenient to introduce the subscripts $o$ and $e$ to label the ordinary and extraordinary mode contributions respectively. This step results in the solutions

$$
\begin{aligned}
\kappa_{o x}= & \pm \sqrt{\varepsilon_{1}-\kappa_{z}^{2}} \\
\kappa_{e x}= & \frac{1}{\varepsilon_{x x}}\left[-\kappa_{z} \varepsilon_{x z} \pm \sqrt{\kappa_{z}^{2}\left(\varepsilon_{x z}^{2}-\varepsilon_{x x} \varepsilon_{z z}\right)-\varepsilon_{1} \varepsilon_{3} \varepsilon_{x x}}\right] .
\end{aligned}
$$

For a waveguide, these equations give the $\kappa_{x} / \kappa_{z}$ relationship for each layer. Since the interest here is in waves that do not attenuate in $z$-direction, $\kappa_{z}$ must be real. Thus it is clear from (8) that for

$$
\kappa_{z}^{2}<\varepsilon_{1} \quad \text { and } \quad \kappa_{z}^{2}\left(\varepsilon_{x z}^{2}-\varepsilon_{x x} \varepsilon_{z z}\right)-\varepsilon_{1} \varepsilon_{3} \varepsilon_{x x}>0
$$

the solutions for $\kappa_{x}$ are real, and for

$$
\kappa_{z}^{2}>\varepsilon_{1} \quad \text { and } \quad \kappa_{z}^{2}\left(\varepsilon_{x z}^{2}-\varepsilon_{x x} \varepsilon_{z z}\right)+\varepsilon_{1} \varepsilon_{3} \varepsilon_{x x}<0
$$

the solution for the ordinary mode will be imaginary, and the solution for the extraordinary mode will be complex. In the guide, these results will determine whether the modes are homogeneous, inhomogeneous or leaky modes. For propagation along the guide, fields that decay away from the surfaces into the semi-infinite media of the substrate and cover are sought. If either or both of these layers are isotropic, then, for guided waves, the first of (10) must be satisfied, and exponential decay will occur. Using the second of (10), for an anisotropic substrate, the decay will not be exponential due to the complex values of the solution for the extraordinary modes given by (8). In this case, solutions are sought which determine that the electric and magnetic fields decay to zero as $x \rightarrow \pm \infty$.

For the specific cases discussed below it is assumed that that the cladding is air and in the anisotropic case $\varepsilon_{1 f}>\varepsilon_{1 s}>\varepsilon_{3 f}>\varepsilon_{3 s}>\varepsilon_{1 c}$. In the hyperbolic film case $\varepsilon_{1 f}>\varepsilon_{1 s}>\varepsilon_{3 f}>\varepsilon_{3 s}>\varepsilon_{1 c}$ and $\varepsilon_{3 f}<0$. Thus, for the constituents of the slab waveguide shown in Figure 3, the necessary conditions for pure hybrid homogeneous modes are: for the film:

$$
\begin{aligned}
& \kappa_{z}^{2}\left(\varepsilon_{x z f}^{2}-\varepsilon_{x x f} \varepsilon_{z z f}\right)+\varepsilon_{1 f} \varepsilon_{3 f} \varepsilon_{x x f}>0 \text { and } \\
& \varepsilon_{1 f}-\kappa_{z}^{2}>0
\end{aligned}
$$

for the anisotropic substrate:

$$
\begin{aligned}
& \kappa_{z}^{2}\left(\varepsilon_{x z s}^{2}-\varepsilon_{x x s} \varepsilon_{z z s}\right)+\varepsilon_{1 s} \varepsilon_{3 s} \varepsilon_{x x s}<0 \text { and } \\
& \varepsilon_{1 s}-\kappa_{z}^{2}<0
\end{aligned}
$$

and for the isotropic cover:

$$
\varepsilon_{1 c}-\kappa_{z}^{2}<0 .
$$

Using (11) and (12) and the definitions in (2), pure homogeneous hybrid waves with real $\kappa_{z}$ can be captured, in terms of $\theta$ and $\phi$, by the following inequalities. 


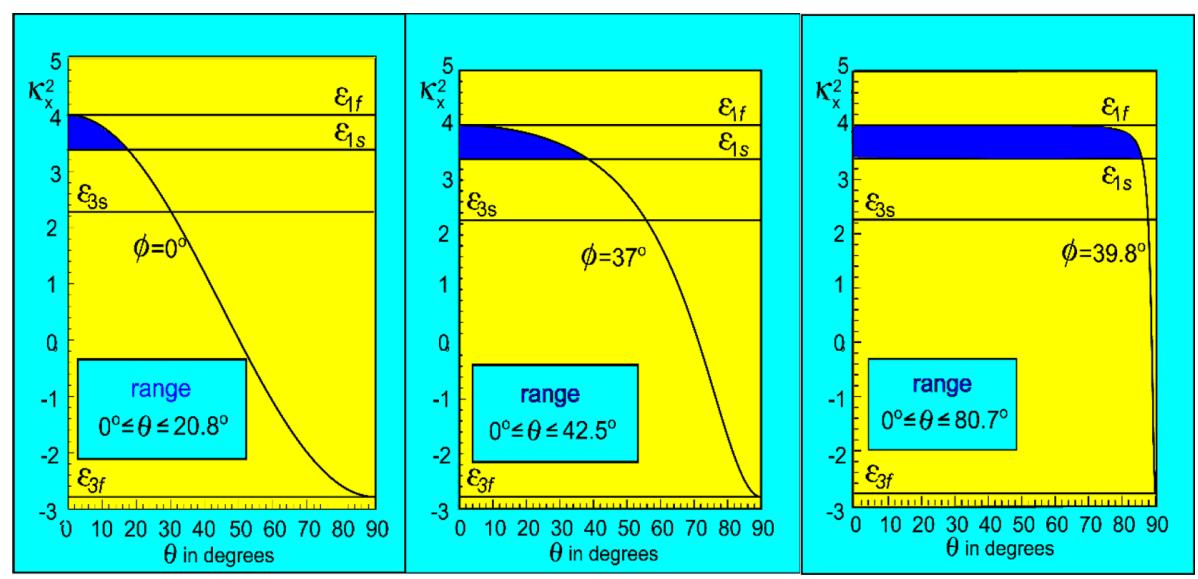

Figure 5. The figure demonstrates the increasing range in $\theta$ over which homogeneous waves occur in a hyperbolic film with $\varepsilon_{3 f}<0$, as $\phi$ increases towards a certain critical value. The dark blue areas show the regions over which pure hybrid homogeneous guided wave are possible.

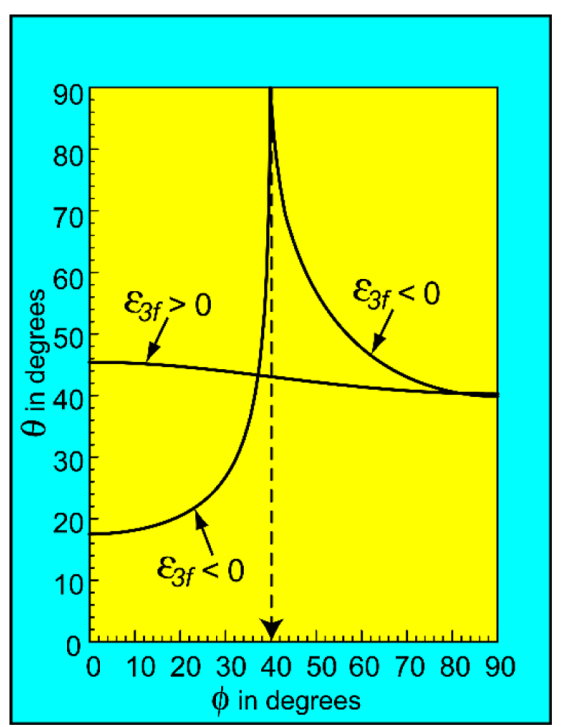

Figure 6. The graphs show the range of $\theta$ over which homogeneous waves can propagate for $\varepsilon_{1 s}=3.3856, \quad \varepsilon_{3 s}=2.25, \quad \varepsilon_{1 f}=4$, $\left|\varepsilon_{3 f}\right|=2.7889$. The range extends from $\theta=0^{\circ}$ up to each of the curves in the figure. As $\phi$ increases, there is little change when $\varepsilon_{3 f}>0$. When $\varepsilon_{3 f}<0$, there is a rapid increase in the range until, at a particular value $\phi=\phi_{\text {crit }}$, homogeneous waves can propagate at all angles of $\theta$ up to $90^{\circ}$. With the parameters given in the text $\phi_{\text {crit }}=39.9^{\circ}$ (shown by the dotted line). The upper right hand curve shows the decreasing range of $\theta$ when $\varphi$ increases beyond $\phi=\phi_{\text {crit }}$.

For the extraordinary mode:

$$
\begin{aligned}
\varepsilon_{3 s} & \frac{\varepsilon_{1 s}+\left(\varepsilon_{3 s}-\varepsilon_{1 s}\right) \cos ^{2} \phi \sin ^{2} \theta}{\varepsilon_{3 s}-\left(\varepsilon_{3 s}-\varepsilon_{1 s}\right) \sin ^{2} \theta \sin ^{2} \phi}<\kappa_{z}^{2} \\
& <\varepsilon_{3 f} \frac{\varepsilon_{1 f}+\left(\varepsilon_{3 f}-\varepsilon_{1 f}\right) \cos ^{2} \phi \sin ^{2} \theta}{\varepsilon_{3 f}-\left(\varepsilon_{3 f}-\varepsilon_{1 f}\right) \sin ^{2} \theta \sin ^{2} \phi}
\end{aligned}
$$

and for the ordinary mode:

$$
\varepsilon_{1 c}<\kappa_{z}^{2}<\varepsilon_{1 f}
$$

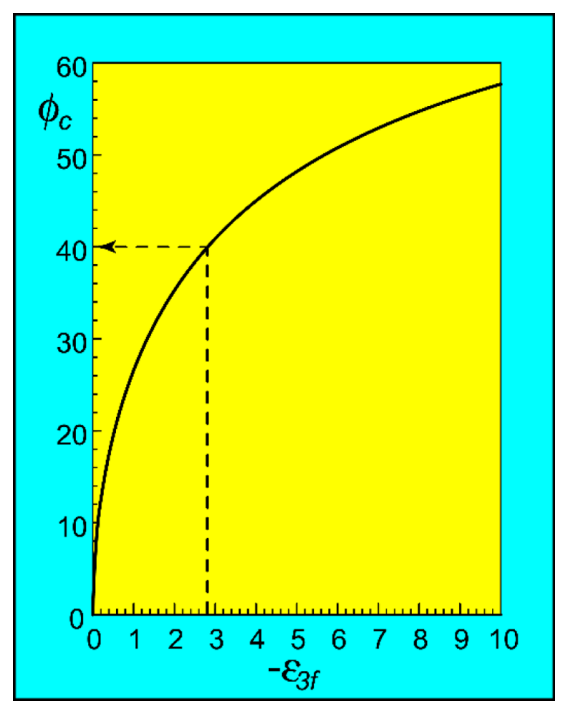

Figure 7. The dependence of the critical angle ( $\phi_{c}$ in the figure) on the value of $\varepsilon_{3 f}<0$, above which the wave number $\kappa_{z}$ is no longer limited by the value of $\varepsilon_{3 f}$ when this is negative. When $\varepsilon_{3 f}>0$ the value of $\kappa_{z}^{2}$ must be less than $\varepsilon_{3 f}$ for propagation of hybrid homogeneous modes. If $\varepsilon_{3 f}<0$, there will be an angle $\phi_{\text {crit }}$ above which there will be propagation of inhomogeneous modes. The arrow in the diagram shows the critical angle when $\varepsilon_{3 f}=-2.7889$.

For a substrate with parameters $\varepsilon_{1 s}=3.3856, \varepsilon_{3 s}=2.25$, and a thin film guide with parameters $\varepsilon_{1 f}=4, \varepsilon_{3 f}=2.7889$, the effect of changing the value of $\phi$ is very small as can be seen in Figure 4. Equations (11) and (12) give the blue curves when $\phi=0^{\circ}$, and the red curves when $\phi=90^{\circ}$. Applying equations (14) and (15) produces the areas in blue from which it can be seen that the change in $\phi$ has little effect on the range of $\theta$ over which pure homogeneous modes occur.

Now the inequalities given in (14) and (15), permitting the existence of various types of waves, are quite general. For a 


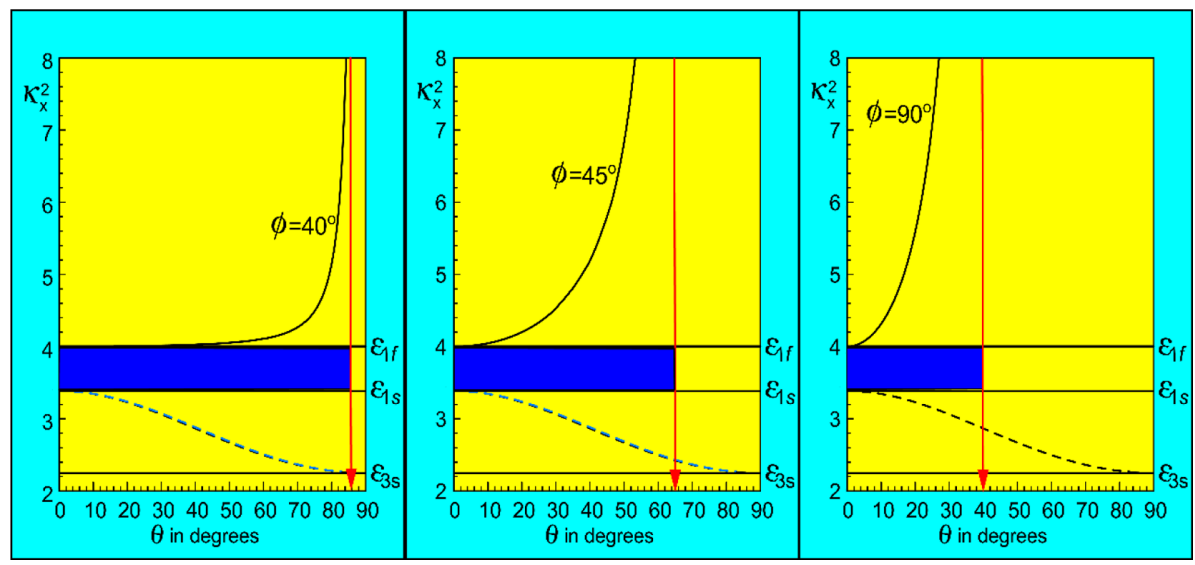

Figure 8. Above critical angle $\phi_{c}$, the normalised wave number $\kappa_{z}$ is no longer limited by the value of $\varepsilon_{1 f}$, but, in the regions above $\varepsilon_{1 f}$ and below the solid curves, the modes are inhomogeneous. The blue areas are the regions where hybrid homogeneous modes occur. The figure shows the decreasing range of $\theta$ over which these modes are possible as $\phi$ increases beyond the value of $\phi_{c}$. The dashed line is the result for the anisotropic substrate where as $\phi$ changes the change in the graph is undetectable on the scale of the figure.

hyperbolic film, where $\varepsilon_{3 f}<0$, the inequality on the right hand side of (14) becomes

$$
\kappa_{z}^{2}<\left|\varepsilon_{3 f}\right| \frac{\varepsilon_{1 f}-\left(\left|\varepsilon_{3 f}\right|+\varepsilon_{1 f}\right) \cos ^{2} \phi \sin ^{2} \theta}{\left|\varepsilon_{3 f}\right|-\left(\left|\varepsilon_{3 f}\right|+\varepsilon_{1 f}\right) \sin ^{2} \theta \sin ^{2} \phi} .
$$

The profound effect of this change can be demonstrated, in detail, by looking analytically at the changes in the regions where the hybrid homogeneous, inhomogeneous and leaky modes can exist. In order to illustrate the specific effect of a negative value for $\varepsilon_{3 f}$, the value $\varepsilon_{3 f}=-2.7889$ will be selected, and the other permittivity values will remain the same. The effect of this change in sign is shown in Figure 5, and contrast greatly from the results shown in Figure 4.

From Figure 5, it is apparent that the pure hybrid homogeneous mode region depends on the point where the curves given by

$$
\kappa_{z}^{2}=\left|\varepsilon_{3 f}\right| \frac{\varepsilon_{1 f}-\left(\left|\varepsilon_{3 f}\right|+\varepsilon_{1 f}\right) \cos ^{2} \phi \sin ^{2} \theta}{\left|\varepsilon_{3 f}\right|-\left(\left|\varepsilon_{3 f}\right|+\varepsilon_{1 f}\right) \sin ^{2} \theta \sin ^{2} \phi}
$$

for the different values of $\phi$ cut the line $\kappa_{z}^{2}=\varepsilon_{1 s}$. These points are given when $\theta=\theta_{\phi}$, where, for $\varepsilon_{3 f}>0$,

$$
\theta_{\phi}=\sin ^{-1} \sqrt{\frac{\varepsilon_{3 f}\left(\varepsilon_{1 f}-\varepsilon_{1 s}\right)}{\left(\varepsilon_{1 f}-\varepsilon_{3 f}\right)\left(\varepsilon_{3 f} \cos ^{2} \phi_{f}+\varepsilon_{1 s} \sin ^{2} \phi_{f}\right)}}
$$

which, as is apparent in Figure 4, decreases, but not very significantly, as $\phi$ changes from $0^{\circ}$ to $90^{\circ}$. For the hyperbolic case, for which $\varepsilon_{3 f}<0$, (17) can be written

$$
\theta_{\phi}=\sin ^{-1} \sqrt{\frac{\left|\varepsilon_{3 f}\right|\left(\varepsilon_{1 f}-\varepsilon_{1 s}\right)}{\left(\left|\varepsilon_{3 f}\right|+\varepsilon_{1 f}\right)\left(\left|\varepsilon_{3 f}\right| \cos ^{2} \phi_{f}-\varepsilon_{1 s} \sin ^{2} \phi_{f}\right)}} .
$$

There is a dramatic difference in the two cases. When $\phi=0^{\circ}$, the ranges of $\theta$ over which pure hybrid homogeneous waves propagate extend to

$$
\theta_{\max }=\sin ^{-1} \sqrt{\frac{\varepsilon_{1 f}-\varepsilon_{1 s}}{\varepsilon_{1 f}-\varepsilon_{3 f}}} \quad\left(\text { for the case where } \varepsilon_{3 f}>0\right)
$$

and

$$
\theta_{\max }=\sin ^{-1} \sqrt{\frac{\varepsilon_{1 f}-\varepsilon_{1 s}}{\left|\varepsilon_{3 f}\right|+\varepsilon_{1 f}}}\left(\text { for the case where } \varepsilon_{3 f}<0\right)
$$

Comparing (19) and (20) it is clear that when $\varepsilon_{3 f}>0^{\circ}$ and $\phi=0^{\circ}$, so that the optic axis is in the $x z$-plane, the range for the anisotropic guide is wider than that for the hyperbolic guide. In the former case, as $\phi$ increases from zero, it is easy to see from (18) that for $\varepsilon_{3 f}>0$, as $\left|\varepsilon_{3 f}\right| \cos ^{2} \phi_{f} \rightarrow \varepsilon_{1 s} \sin ^{2} \phi_{f}$, the range of $\theta$ over which pure hybrid homogeneous modes can exist decreases, but only by a relatively small amount (Figures 4 and 6). In contrast to this case, when $\varepsilon_{3 f}<0$ and $\phi$ increases from zero, the range increases rapidly until, as $\phi$ approaches a certain critical value $\phi_{\text {crit }}$, given by

$$
\phi_{\text {crit }}=\sin ^{-1} \sqrt{\frac{\left|\varepsilon_{3 f}\right|}{\left|\varepsilon_{3 f}\right|+\varepsilon_{1 f}}},
$$

pure hybrid homogeneous modes can exist for all values of the angle $\theta$ from $0^{\circ}$ to $90^{\circ}$ (Fgures 5 and 6). Note that as $\phi$ approaches the value $\phi_{\text {crit }}$, the change in the value of $\theta_{\max }$ becomes very rapid so that very small changes in the orientation of the optic axis has a profound effect, which could have important consequences for the design and manufacture of hyperbolic guides.

It is apparent from Figure 6 that for the case where $\varepsilon_{3 f}>0$, the critical value of $\phi$ has no meaning. It should be noted here that the critical value also depends crucially on the value of $\varepsilon_{3 f}$ as shown in Figure 7.

A second important major difference between the usual anisotropic case and the hyperbolic case is that in the latter modes can propagate at values of $\kappa_{z}^{2}>\varepsilon_{1 f}$. However, in this region the condition for the ordinary wave in equation (2), 
namely, $\varepsilon_{1 f}-\kappa_{z}^{2}>0$ is no longer satisfied, and the waves are inhomogeneous. These waves and the associated leaky waves will be examined in detail in a future paper.

As the angle $\phi$ increases beyond the value of $\phi_{c}$, the range of $\theta$ over which pure hybrid homogeneous waves propagate decreases again and is given by:

$$
0<\theta<\sin ^{-1} \sqrt{\frac{\left|\varepsilon_{3 f}\right|}{\left(\left|\varepsilon_{3 f}\right|+\varepsilon_{1 f}\right) \sin ^{2} \phi}}
$$

Figure 8 demonstrates this decreasing range of range of $\theta$ over which these modes propagate as $\phi$ increases from a value just above $\phi_{c}$ to the angle $\phi=90^{\circ}$.

The above analysis shows, for a particular data set, how varying the position of the optic axis leads to interesting conclusions about the regions of existence of different forms of waves. In particular it shows how varying the orientation of the optic axis creates, or closes, windows of opportunity to propagate the kind of waves that are certain to be part of upcoming devices. In order for a meta-device to operate in a homogeneous wave window it is important first of all to conduct an investigation into how the orientation angle $\phi$ can be made to influence the wave outcomes. The analysis also introduces the existence of inhomogeneous waves for values of the normalised wave-number that lie above the maximum possibilities for the anisotropic guide with a permittivity tensor with all positive elements.

\section{Conclusions}

As opposed to what is revealed in the limited literature on optic axis orientations in metamaterials, the general theory given here shows the advantages of orienting the optic axis in carefully defined directions.

Through examining the dispersion relations for hyperbolic waveguides, this paper emphasises that the orientation of the optic axes is a significant determinant in the resulting optical properties. In fact, whereas for conventional anisotropic waveguides, homogeneous propagating waves occur over a limited range of the polar angle $\theta$, a range that varies only a little with the azimuthal angle $\phi$, we have shown that for a hyperbolic guide, a critical angle $\phi_{c}$ exists, at which value the guided waves are homogeneous for all values of $\theta$ between $0^{\circ}$ and $90^{\circ}$. This has significant implications for meta-waveguide designs in that it implies that, in the hyperbolic case, generation of propagating modes can be more easily achieved, and the resulting structures are more tolerant to optic axis misalignment.

The high sensitivity of the mode propagation constant to azimuthal orientation of the optic axis could also be transformative for meta-guided wave technology. Small external perturbations, such as the application of mechanical pressure, or temperature, can result in changes to the effective orientation of the guide/substrate axes. These in turn, will result in modes being cut off, or allowed to propagate, opening up potential for sensor applications. Indeed, electro-optic control over the orientation of the optic axis in a hyperbolic guide could lead the way to a communications technology based on active mode control.

Also, we note the implication of our work for transformation designs that exploit the orientation of the optic axis of anisotropic media, as described in [13]. In that work, pixels of anisotropic media, where the orientation of the optic axis alternated between pixels, were shown to produce an effective transmission medium capable of a variety of transformation devices, including a wave expander and a source shifter. Our work suggests that a greater range of applications is possible through the use of pixelated hyperbolic media.

\section{References}

1. E. Bartholin, Experimenta crystalli islandici disdiaclastici quibus mira \& insolita refractio detegitur, Copenhagen ("Hafniæ"), Denmark, 1669.

2. L. Ferrari, C. Wu, D. Lepage, X. Zhang, Z. Liu, Progress in Quantum Electronics 40 (2015) 1.

3. D.C. Kay, in Schaum's outline of theory and problems of tensor calculus, McGraw-Hill, New York, 1988.

4. E. Starodubtsev, Proc. SPIE 7353 (2009) Metamaterials IV 7353OA.

5. S.M. Hashemi, I.S. Nefedov, M. Soleimani, Photonics Letters of Poland 5 (2013) 72.

6. I.S. Nefedov, C.A. Valagiannopoulos, S.M. Hashemi, E.I. Nefedov, Scientific Reports 3 (2013) 2662.

7. A. Knoesen, T.K. Gaylord, M.G. Moharam, Journal of Lightwave Technology 6 (1988) 1083.

8. D.P. Gia-Russo, J.H. Harris, Journal of the Optical Society A 63 (1973) 138.

9. D. Marcuse, IEEE Journal of Quantum Electronics QE-14 (1978) 736.

10. D. Marcuse, I.P. Kaminow, IEEE Journal of Quantum Electronics QE-15 (1979) 736.

11. K.E. Ballantine, P.R. Eastham, J.F. Donegan, Physical Review A 90 (2014) 013803.

12. J.A. Fleck, M.D. Feit, Journal of the Optical Society A 73 (1983) 920.

13. Z. Liang, X. Jiang, F. Miao, S. Guenneau, J. Li, New Journal of Physics 14 (2012) 103042.

Cite this article as: Boardman $\mathrm{AD}$, Egan $\mathrm{P} \& \mathrm{McCall} \mathrm{M}$ : Optic axis-driven new horizons for hyperbolic metamaterials. EPJ Appl. Metamat. $2015,2,11$. 